schen, erwähnen, oder etwa die Risiken der Unterernährung beim Anbau von für den Export bestimmten landwirtschaftlichen Produkten.

- Denn in dieser Situation, die gekennzeichnet ist durch den Hunger, die Nichtbefriedigung von Grundbedürfnissen, wie zum Beispiel Wohnung und Bekleidung, oder auch durch mangelnde Hoffnung und fehlendes Selbstvertrauen, steht die Medizin gleichsam vor einer Sisyphusarbeit: das Elend zerstört in dem Masse die Menschen, in welchem die Medizin zu helfen sucht. Solange nicht die allgemeine Verbesserung der Lebensbedingungen für alle Menschen als erstes Entwicklungsziel postuliert wird, werden wir immer das Gefühl haben, Hautschürfungen auf einem brandigen Körper zu pflegen.

Bevor ich meine Rede beende, möchte ich noch kurz die praktischen Folgerungen aufzeigen, welche wir aus den vorgängigen Überlegungen und aus der Analyse der gemachten Erfahrungen ziehen. Es genügt nicht, dass die schweizerische Entwicklungszusammenarbeit da und dort ein Spital oder ein Dispensarium errichtet, so nützlich diese auch sein können. Sie muss sich vor allem an der Errichtung eines kohärenten Systems - durch das betreffende Entwicklungsland - beteiligen. Ausgehend vom komplementären Charakter unseres Beitrages müssen wir analysieren, auf welcher Ebene die ausländische Hilfe sich als am nützlichsten erweisen kann. Es gibt Länder, denen es bereits gelungen ist, genügend Personal für die Pflege in den Dörfern bereit- zustellen, und die somit eine Unterstützung von aussen für Verbesserungen auf höchstem wissenschaftlichen und technischem Niveau suchen. Andere wünschen die Prioritätsordnung, die sie in früheren Jahren angenommen hatten, umzukehren und benötigen deshalb finanzielle Mittel und Personal, um das Gesundheitswesen vermehrt auf die Dörfer auszudehnen. Was in diesem wie auch in allen anderen Bereichen der Entwicklungszusammenarbeit zählt, ist die Bereitschaft der reichen Länder zur Beseitigung der Hindernisse, an denen sich unsere Partner in der Dritten Welt stossen, beizutragen.

Die ausserordentlichen Fortschritte der Wissenschaft und ihre Anwendung im Kampf gegen Krankheit und Tod ziehen ein doppeltes Risiko nach sich: Die Kranken könnten als Kinder betrachtet werden, die man in Obhut nehmen muss, oder gar als Maschinen, die zu reparieren sind.

Indes, wir wissen heute, dass Gesundheit nur möglich ist, wenn sie von allen gewünscht und bewusst angestrebt wird. Und so wie sich der Kranke an seiner Heilung beteiligen muss, muss jeder einzelne an der Entwicklung seiner Gesellschaft teilnehmen.

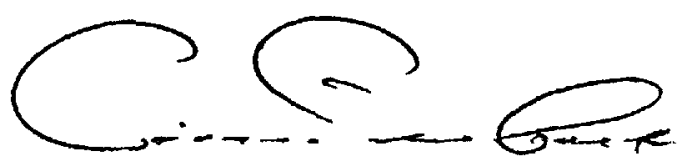

Pierre Aubert, Bundesrat

\title{
Editorial
}

\section{Coopération médico-sanitaire au développement: malade, convalescente ou en bonne santé?}

\section{J. Martin ${ }^{\top}$}

Le présent numéro de Médecine sociale et préventive pose plus de questions qu'il ne donne de réponses. Après que les Nations Unies eurent proclamé une première Décennie du développement, nous sommes maintenant au terme de la seconde, et l'évaluation du chemin parcouru et des perspectives d'avenir reste bien peu claire et pas souvent vrai-

'Médecin cantonal adjoint, Service de la santé publique, $\mathrm{CH}$ 1001 Lausanne.

Ex-médecin (santé familiale), Bureau régional de l'Asie du Sud-Est de l'OMS, New Delhi.

Ex-Lecturer in Maternal and Child Health, School of Public Health, University of North Carolina, Chapel Hill, N.C., USA. ment optimiste. On parle de «stagflation» dans les pays industrialisés et de «croissance sans développement» dans le tiers monde; souvent, il y a de sérieuses raisons de penser que, dans les pays défavorisés, le lot de la majorité est en fait en train de se péjorer.

Le fossé entre ceux qui ont et ceux qui n'ont pas (the haves and the have-nots) s'agrandit chaque jour; que cette expression soit devenue un cliché banal n'en atténue pas la gravité. Et c'est une évidence qui permet, objectivement, aux détracteurs des entreprises de coopération technique ou simplement aux sceptiques de nous proposer après 
Hugo de «traire notre vache et vivre en paix...». A un autre niveau d'analyse, à l'aide d'une dialectique de virtuose et d'une grande érudition, Hlich et son groupe de Cuernavaca (voir article de V.Borremans) décrivent les aberrations monopolistes et professionnalisantes de l'envahissant modèle occidental, et appellent de leurs vœux la société conviviale où chaque individu redeviendra beaucoup plus polyvalent et, par voie de conséquence, autosuffisant, indépendant des biens et des services manufacturés par d'autres ou par des machines-outils informatisées. L'ennui (c'est l'humble réflexion de l'auteur de ces lignes, qui par ailleurs reste impressionné par les thèses illichiennes), l'ennui c'est que le groupe du CIDOC ne donne guère d'indications, à ceux dont la tendance est de vouloir «faire quelque chose», sur les voies et moyens praticables d'éliminer ou au moins d'aménager les excès de l'évolution que nous vivons. Et il se défend de vouloir le faire.

Dans ce contexte, on relira avec intérêt les remarques de Benjamin Franklin sur «les sauvages de l'Amérique du Nord». A vrai dire, elles appellent la question de savoir si, sur le plan conceptuel, nous avons fait un progrès en deux siècles (et peut-être en quarante siècles?). Sommes-nous condamnés à continuellement réinventer la roue?

Alors, la coopération au développement... Très malade? Irréversiblement malade?

A côté de ceux pour lesquels la tâche est sans espoir ou sans valeur, il y a ceux qui pensent nécessaire de faire quelque chose. Qui notent en particulier que ce n'est pas la suppression de l'entraide technique qui va empêcher les effets indésirables de la civilisation industrielle (qu'elle vienne de l'Occident ou d'ailleurs) de se faire sentir de façon de plus en plus marquée et, il faut bien le dire, corrosive souvent, dans les pays à structure pour l'instant plus traditionnelle. Leisinger et Gerster le relèvent dans leur article sur une des nations de la planète qui est restée le plus longtemps très isolée du reste du monde, et il est vraisemblable que tous ceux qui contribuent à ce numéro se sont une fois ou l'autre accordé cette justification (correcte à notre avis). Ridet et Selby rappellent que, s'agissant de la santé psychosociale et aussi physique des collectivités, dans les pays pauvres qui ont un potentiel dans ce sens, le développement du tourisme amène son cortège de maux, dont la prostitution est un des plus visibles mais n'est pas le seul. Pradervand présente un effort, couronné de succès pour l'instant, qui cherche à combattre le cosmopolitisme de bas niveau qui caractérise la grande majorité de ce que disséminent les mass media dans le tiers monde. Et Cadotsch, après avoir décrit un programme qui peut être considéré à divers titres comme une réussite, doit rapporter qu'une nouvelle orientation politique fait revenir le pays concerné dix ans en arrière (avant le moment où un groupe de militaires motivés décida de prendre des mesures effectives pour tenter d'intégrer la majorité indienne du pays à la dynamique nationale).

Mais rien n'est jamais absolument juste ou absolument faux ( «jamais et toujours n'existent pas en médecine», disaient nos professeurs). Nous vivons dans un monde où le Suisse sortant de son pays découvre que certains problèmes, beaucoup de problèmes, n'ont pas vraiment de bonne solution, mais seulement des mauvaises ou des moins mauvaises (par contraste avec ce qu'on peut presque croire chez nous). Pourquoi alors serait-il vain de vouloir faire sa part, aussi peu encourageantes que soient certaines constatations? Les auteurs contribuant à ce fascicule et les organisations qui les ont envoyés ou, qui d'une autre manière, apportent une collaboration aux pays en développement, ont décidé qu'il valait la peine de s'engager activement.

Une des lignes de force de ce numéro est sans doute la façon dont tant d'individus et de groupes décrivent la nécessité dans laquelle ils se sont trouvés de changer. Nécessité d'élaborer, au cours d'un processus ardu, parfois déchirant, une nouvelle conception de leur rôle, de ce qu'ils pouvaient apporter à leurs homologues (et hôtes); conception qui tienne compte de la réalité et pas surtout de louables sentiments ou d'une morale. Ils (et elles) ont tous changé...; on ne revient pas d'un «tour» de coopération au développement sans avoir dû effectuer un réaménagement majeur de ses idées et de ses priorités. On peut dire en passant que, sans doute possible, il y a là un effet positif des efforts de coopération technique des dernières décennies. Pour avoir côtoyé souvent, au cours de huit ans et sur trois continents, des volontaires du Corps de la paix américain (par exemple), il m'est souvent arrivé de penser: «Je ne suis pas à même d'évaluer le progrès dont ils ont fait bénéficier les régions où ils sont allés, mais ils ont sûrement beaucoup apporté à leur propre pays» (si l'on prend la liberté de citer une organisation, ce n'est pas pour la singulariser, et sûrement pas pour dire qu'elle serait plus criticable que d'autres, mais seulement parce qu'elle vient à l'esprit). Le sérieux problème ici est bien sûr que cet exemple constitue un gain pour le pays «donneur», alors qu'il est abondamment répété que les bénéfices de la coopération doivent aller d'abord et surtout aux pays en développement. Faut-il rappeler que les constatations à cet égard sont cependant souvent ambivalentes?

Pour que l'effort porte des fruits notables dans les pays partenaires, il est impératif de se pénétrer et d'observer un certain nombre de règles que beaucoup des textes de ce fascicule décrivent, sans éviter des répétitions (qu'on veuille bien accepter que, dans le cas particulier, bis repetita placent). S'il fallait en choisir une parmi elles, ce serait probable- 
ment «écouter, écouter, et écouter» (avec les oreilles, avec les yeux, de toutes les façons possibles).

Nous parlons de règles, mais il ne s'agit pas seulement d'appliquer des techniques, aussi participatives ou «behaviorales» soient-elles. Ce sont surtout les attitudes qui feront la différence, attitudes qui sont aussi déterminantes d'un «côté» que de l'autre. Lors d'une réunion (dite sur les ressources extra-budgétaires) tenue en novembre 1978 à l'OMS, à Genève, suite à la Conférence d'Alma Ata, le professeur T. A. Lambo, psychiatre nigérian, ancien vice-chancelier de l'Université d'Ibadan et actuel directeur général adjoint de l'OMS, a parlé de façon très vigoureuse de la nécessité impérative où nous nous trouvons de modifier certaines habitudes jusqu'ici immuables; habitudes de courtoisie de circonstance quelles que soient les actions (et les attitudes) des uns et des autres. Nous devons, a dit le Dr Lambo, cesser de considérer les responsables nationaux dans les pays du tiers monde comme des «cottonwool babies». Nous ne devons pas craindre d'être «brutalement francs» entre nous, afin d'identifier précisément les obstacles et d'étudier résolument les aménagements nécessaires, de part et d'autre. Si un tel discours eût pu être difficilement acceptable suivant son origine, il est vrai que le professeur Lambo est dans une situation qui lui permet de le faire.

Alors, la coopération au développement... Convalescente? En voie de rétablissement?

Quelques mots sur les communications rassemblées dans ce numéro. Il nous semble qu'elles constituent un éventail assez varié et démonstratif de la part que prend notre pays à la coopération médicosanitaire au développement (nous n'oublions pas par ailleurs que nous devons quelques articles à des collègues étrangers, auxquels nous disons notre gratitude). Certaines décrivent des programmes qui se sont développés au cours de près de vingt ans, comme le travail de l'Institut tropical suisse en Tanzanie; des organisations présentées ici ont derrière elles toute une tradition de travail outre-mer, mais d'autres sont beaucoup plus jeunes. Certaines sont officielles, d'autres sont privées. Des articles décrivent comment les choses se passent sans nous (contribution de Choffat notamment, ainsi que de multiples éléments sur l'importance du contexte socio-culturel, dans des articles présentant par ailleurs des programmes d'«intervention» dans cette réalité); plusieurs sont dans l'orbite des orientations sur lesquelles on a relativement récemment mis l'accent, comme l'éducation de masse et la participation (Pradervand, Projet «Vivamos mejor» cité par Abelin et Zehnder). Beaucoup vont dans le sens des soins de santé primaires, «consacrés» en septembre 1978 par la
Conférence d'Alma Ata et qui sont une des composantes essentielles de ce que d'aucuns appellent la «révolution mahlérienne», qu'a connue l'OMS au cours des dernières années.

Nous savons tout spécialement gré au $D r$ H. Mahler, directeur général de l'OMS, de nous avoir fait l'honneur de préfacer ce numéro et de présenter certaines des considérations qui sont à la base de l'action actuelle de son organisation. Nous sommes heureux aussi du privilège de publier l'allocution qu'a donnée en juin 1978 le conseiller fédéral Pierre Aubert lors de la Conférence annuelle de la Direction de la coopération au développement et de l'aide humanitaire du Département politique fédéral, aux destinées duquel il préside. Après les éditoriaux viennent deux contributions d'origine américaine, l'une historique et l'autre moderne, que nous avons évoquées plus haut; puis des articles mettant l'accent sur la planification, la gestion et le financement des services de santé, moins prestigieux que la "grande» médecine mais d'immense importance (articles de Piot et Mach, tous deux de l'OMS).

Un chapitre «Formation et recherche» présente des contributions de l'Institut tropical suisse (Freyvogel et Degrémont), puis deux efforts de recherche sur le terrain, en Afrique, dont le souci est d'avoir aussi vite que possible des conséquences opérationnelles utiles aux populations concernées (Rougemont et coll.; Ridet et Selby). La partie «Soins de santé primaires et agents de santé communautaires» commence par des considérations fondamentales, basées sur une expérience pratique bien documentée, d'un Suisse qui se trouve depuis des années de l'autre côté de l'Atlantique (Habicht). Cadotsch et Gachoud, dont les communications suivent, sont restés moins longtemps dans l'hémisphère Ouest, tout en travaillant aussi à la formation et à l'utilisation d'agents de santé primaires, les auxiliaires volontaires de santé de l'Altiplano péruvien. Puis Baur et Ch. Raeber discutent, sur la base d'expériences en Afrique centrale, le besoin de «sortir de l'hôpital» et de mettre en œuvre une véritable animation sanitaire (ce qui ne va pas sans difficultés). Choffat, lui, a vécu au contact très proche d'un système médical traditionnel dans le Grand Sud marocain.

L'action à large échelle, notamment pas les mass media, peut être utilement complémentaire de ce qui se fait sur le terrain. «Famille et Développement» par exemple (article de Pradervand) est une entreprise d'éducation au développement dont les messages soutiennent vigoureusement l'action de ceux qui œuvrent localement dans les secteurs pédagogique, sanitaire, social, agricole, etc., et devrait potentialiser cette action. L'Année internationale de l'Enfant aura elle aussi, on le souhaite, un impact en profondeur. 
Dans un dernier volet (mais pas le moindre) sont présentées les activités entreprises par diverses organisations. Le FISE/UNICEF tout d'abord, par son directeur exécutif adjoint, le $\operatorname{Dr} C$. A. Egger, un de nos compatriotes, et la Direction de la coopération au développement $\mathrm{du}$ gouvernement suisse (Wilhelm et Zanolli). Puis six organisations privées, cinq dont l'objectif premier est l'action pratique dans le cadre de projets de développement, Frères des Hommes (Mongin), Helvetas (Leisinger et Gerster), Medicus Mundi (Widmer), Swissaid (Schnellmann), et une orientée principalement vers la recherche, la Fondation Nestlé (Aebi et coll.).

Tout cela permet-il de dire que tout va pour le mieux dans la coopération technique issue de Suisse? Il serait un peu présomptueux de le prétendre. Entre autres choses parce que quantitativement la contribution de notre pays reste certainement insuffisante. Sur le plan qualitatif, l'auteur de cet éditorial a la témérité de penser que, dans l'ensemble, les communications de nos collègues donnent une impression saine; que les remises en question, les approches et les actions pratiques actuelles vont largement dans le bon sens. Sur cette base, on peut espérer que ce fascicule de Médecine sociale et préventive apportera sa pierre à l'effort d'information et de sensibilisation si nécessaire dans les pays industrialisés en ce qui concerne une véritable action conjointe (dans le même sens!) avec ceux avec qui, quoi qu'on fasse, nous sommes dans la même nacelle (le «Spaceship Earth»).

Les connaissances, les compétences et les attitudes à disposition apparaissent honorables. Manquent des ressources. Serons-nous assez convaincants pour en obtenir plus, de façon que l'effort de la Suisse se rapproche de ceux relativement bien plus importants d'autres petits pays industrialisés, de l'Europe du Nord notamment (avec lesquels par ailleurs nous avons passablement de points communs). En janvier dernier, le Conseiller fédéral Aubert, dans un exposé fait à Zurich devant un institut de recherches internationales, soulignait le rôle marquant que peuvent jouer les petits pays, sur le plan diplomatique par exemple, et même sur le plan militaire. Il est tout à fait imaginable que, dans les années qui viennent et si la collectivité accepte d'y mettre le prix, la Suisse joue un rôle nettement plus substantiel que par le passé dans le domaine de la coopération.

Pour que, dans l'avenir, le diagnostic qu'on peut poser sur la coopération au développement soit plus proche de la «bonne santé», de la pleine santé.

\section{Editorial}

\section{Entwicklungszusammenarbeit im Gesundheitswesen: krank, rekonvaleszent oder gesund?}

\section{J. Martin ${ }^{1}$}

Das vorliegende Heft von «Sozial- und Präventivmedizin» stellt mehr Fragen, als es Antworten gibt. Nachdem die Vereinigten Nationen ein Jahrzehnt der Entwicklung ausgerufen hatten, befinden wir uns jetzt am Ende des zweiten, und die Beurteilung des zurückgelegten Weges bleibt ziemlich unklar und oft nicht sehr optimistisch. Man spricht von «Stagflation» in den industrialisierten Ländern und von «Wachstum ohne Entwicklung» in der Dritten Welt; oft gibt es ernsthafte Gründe für die Annahme, dass sich in den benachteiligten Ländern das Los der Mehrheit der Bevölkerung verschlechtert.

1 Dr méd., médecin cantonal adjoint, Service de la santé publique, $\mathrm{CH}-1001$ Lausanne.
Die Gegner der Entwicklungshilfe und die Skeptiker schlagen uns vor, der Devise Hugos $\mathrm{zu}$ folgen, «unsere Kuh zu melken und in Frieden zu leben». Auf einer anderen Ebene der Analyse beschreiben Illich und seine Gruppe von Cuernavaca die Irrwege des aufdringlichen, auf alles übergreifenden westlichen Entwicklungsmodells und rufen nach einer Gesellschaftsform des friedlichen $\mathrm{Zu}$ sammenlebens. In diesem Zusammenhang wird man mit Interesse die Bemerkungen Benjamin Franklins über «die Wilden Nordamerikas» wieder lesen. Haben wir seither in konzeptueller Hinsicht Fortschritte gemacht?

Alle diejenigen, welche der Ansicht sind, dass etwas unternommen werden soll, betonen aber, dass die 